\title{
A Bayesian Estimation Procedure of Reliability Function for Lifetime Distributions
}

\author{
VASILIY KRIVTSOV ${ }^{1}$ and MICAHEL FRANKSTEIN \\ Department of Reliability Analytics \\ The Ford Motor Company, Dearborn, MI, USA
}

(Received on November 21, 2016 and Revised on February 28, 2017)

\begin{abstract}
This paper is a sequel to [1], wherein we proposed a simple procedure to construct the joint prior and posterior distributions of Weibull parameters based on the underlying reliability function estimates in two time cross-sections. In this paper, we extend the procedure in three aspects: a) the prior data can now be taken in terms of a simple probability paper plot, b) the posterior now includes not only posterior estimates of distribution parameters, but also the posterior estimate of the underlying reliability function along with the respective credibility intervals, and c) we show that the proposed procedures can be applied to any parametric lifetime distribution, not necessarily limited to the locationscale family.
\end{abstract}

Keywords: Bayesian estimation, credibility bounds, joint prior distribution

\section{Introduction}

Bayesian estimation of parametric reliability functions has been considered by many authors. A critical first step is the specification of the prior distribution of the reliability function parameter(s). Some authors omit this step and treat it as a given, i.e., the (joint) prior distribution of parameter(s) is assumed to be known. A few authors do discuss procedures of constructing the (joint) prior distribution based on expert estimates or life test data. Under the assumption of independence, one can specify marginal priors of individual distribution parameters, thus arriving to their joint prior. For example, Canavos \& Tsokos [2] assume independent priors for the shape (uniform) and scale (inverse gamma) parameters of the Weibull distribution. Meeker \& Escobar [3] discuss constructing the joint prior distribution of the Weibull distribution based on the Weibull shape parameter and a certain quantile of the underlying CDF. They make a point that in case of heavy censoring (which is often the case in reliability data analysis), specifying the shape parameter and an appropriately chosen quantile of the underlying distribution would be more robust than specifying individual priors for the shape and the scale parameters.

Kaminskiy \& Krivtsov [1] proposed a simple procedure to construct the joint prior distribution of Weibull parameters based on the estimates of the underlying reliability function in two time cross-sections. The outcome of the procedure was the joint prior distribution of Weibull parameters as well as the posterior point estimate of the underlying reliability function at any time of interest. The procedure turned out to be quite useful in 
practical applications. As of 2016, it has been cited in over 70 publications [5] and was implemented in the 2013 release of the $\mathrm{JMP}^{\circledR}$ reliability analysis software package by the SAS Institute [6]. It must be mentioned that the procedure discussed in [3] is also implemented in the same package as an alternative method of specifying the prior.

In this paper, we extend the procedure proposed in [1] in three aspects: a) the input (prior) data can be taken in the form of a simple probability paper plot, b) the output includes not only posterior estimates of the distribution parameters, but also the posterior estimate of the underlying reliability function along with the respective credibility intervals, and c) we show that the proposed procedures can be applied to any parametric lifetime distribution. These extensions make the procedure much more usable for practical engineering applications.

\section{Proposed approach}

Without loss of generality, consider a two-parameter lifetime distribution with the cumulative distribution function $F(t ; \alpha, \beta)$, wherein $\alpha$ and $\beta$ are the scale and the shape parameters, respectively.

A penultimate step in the Bayesian estimation of the reliability function is the posterior joint density of the aforementioned parameters, which is given by:

$$
\pi(\alpha, \beta)=\frac{\pi_{0}(\alpha, \beta) L(\text { data } \mid \alpha, \beta)}{\iint \pi_{0}(\alpha, \beta) L(\text { data } \mid \alpha, \beta) d \alpha d \beta}
$$

where $\pi(\alpha, \beta)$ and $\pi_{0}(\alpha, \beta)$ - the posterior and prior joint PDF of the parameters distribution, respectively; $L-$ the likelihood function relative to current data.

Since the denominator in (1) does not depend on parameters $\alpha$ and $\beta$ (as it integrates to a constant), it is sufficient to estimate only the numerator. Assume that the prior data about parameters $\alpha$ and $\beta$ is available in a form of a random sample from the underlying lifetime distribution:

$$
\left(t_{1}, \delta_{1}\right),\left(t_{2}, \delta_{2}\right),\left(t_{3}, \delta_{3}\right), \ldots,\left(t_{n}, \delta_{n}\right)
$$

where $t_{i}$ - observed lifetime, $\delta_{i}$ - censoring indicator, such that $\delta_{i}=1$ if $t_{i}$ is a complete observation or $\delta_{i}=0$ if $t_{i}$ is a right-censored observation.

Further assume that current data about parameters $\alpha$ and $\beta$ are available in a form of another random sample:

$$
\left(T_{1}, \gamma_{1}\right),\left(T_{2}, \gamma_{2}\right),\left(T_{3}, \gamma_{3}\right), \ldots,\left(T_{m}, \gamma_{m}\right),
$$

where $T_{i}$ - observed lifetime, $\gamma_{i}$ - censoring indicator.

The following bootstrap simulation procedure is proposed to estimate $\pi_{0}(\alpha, \beta) L($ data $\mid \alpha, \beta)$ as well as the posterior reliability function.

Random resampling with replacement from sample (3) is performed $n$ times. A sample is generated at each step $i$ as: 


$$
\left(t_{1_{i}}, \delta_{1_{i}}\right),\left(t_{2_{i}}, \delta_{2_{i}}\right),\left(t_{3_{i}}, \delta_{3_{i}}\right), \ldots,\left(t_{n_{i}}, \delta_{n_{i}}\right)
$$

Distribution parameters are estimated using the maximum likelihood procedure:

$$
\left(\widehat{\mathbf{a}_{1}}, \widehat{\boldsymbol{\beta}_{1}},\right)=\arg \max _{\mathbf{a}_{\mathrm{i}}, \mathbf{b}_{\mathrm{i}}} \operatorname{Ln}\left(L\left(\left(\mathbf{t}_{\mathbf{i}}, \boldsymbol{\delta}_{\mathbf{1}_{\mathrm{i}}}\right),\left(\mathbf{t}_{\mathrm{z}_{\mathrm{i}}}, \boldsymbol{\delta}_{\mathbf{2}_{\mathrm{i}}}\right), \ldots,\left(\mathbf{t}_{\mathrm{n}_{\mathrm{i}}}, \boldsymbol{\delta}_{\mathbf{n}_{\mathrm{i}}}\right)\right)\right)
$$

where the likelihood function $L$ is defined as:

$$
L=\prod_{\mathrm{j}} f\left(\mathrm{t}_{\mathrm{j}}, \mathrm{a}, \beta\right)^{\delta_{\mathrm{j}}}\left(1-F\left(\mathrm{t}_{\mathrm{j}}, \mathrm{a}, \beta\right)\right)^{1-\delta_{\mathrm{j}}}
$$

Then, the posterior likelihood function can be calculated as:

$$
L P_{i}=L\left(\widehat{\mathrm{a}_{1}}, \widehat{\beta_{1}},\left(T_{1}, \gamma_{1}\right),\left(T_{2}, \gamma_{2}\right),\left(T_{3}, \gamma_{3}\right), \ldots,\left(T_{m}, \gamma_{m}\right)\right.
$$

The sample reliability function is:

$$
S_{i}=1-F\left(t ; \widehat{\alpha_{1}}, \widehat{\beta_{1}}\right)
$$

The posterior joint distribution of parameters is estimated as:

$$
\pi_{0}(a, \beta) L(\text { data } \mid a, \beta)=\sum_{i} 1_{\widehat{1_{1}} \leq a, \widehat{\beta_{1}} \leq \beta}\left(\widehat{\mathrm{a}_{1}}, \widehat{\beta_{1}}\right) L P_{i} / \sum_{i} L P_{i}
$$

The posterior reliability function $R(S, t)$ is obtained as:

$$
\boldsymbol{R}(\boldsymbol{S}, \boldsymbol{t})=\sum_{\boldsymbol{i}} \mathbf{1}_{S_{i} \leq S}\left(\boldsymbol{S}_{\boldsymbol{i}}\right) / \boldsymbol{n}
$$

The lower $L C L_{S}$ and upper $U C L_{S}$ two-sided $100 \%(1-\alpha)$ credible limits are found as those satisfying the following equations:

$$
\begin{aligned}
& R\left(L C L_{S}, t\right)=\alpha / 2 \\
& R\left(U C L_{S}, t\right)=1-\alpha / 2
\end{aligned}
$$

\section{Numerical Example}

In this section, the Weibull distribution is used as an example, but the procedure being proposed can be extended to any parametric lifetime distribution (not limited to the location-scale family). In Figure 1, consider a Weibull probability plot of a random sample as in (2) with 39 complete and 11 censored failure times. The MLE estimates of the shape and scale parameters of this sample are $\hat{\beta}=1.7, \hat{\alpha}=43.8$, respectively.

As discussed in Section II, we obtain bootstrap samples (4) of the same size as the original sample - see Figure 2. To each bootstrap sample, we fit a Weibull distribution, thus obtaining a pair of Weibull parameters (5). By repeating the sampling procedure $n$ times, we obtain $n$ pairs of Weibull parameters, on which their joint distribution is constructed.

Figure 3 shows the joint prior distribution of Weibull parameters constructed based on $n=10,000$ bootstrap samples from the sample in Figure 1 . The highest prior density corresponds to $\alpha=50.9$, and $\beta=2.16$.

Using the following Weibull sample, see Figure 4, as the current data (3) we obtain the joint posterior distribution of Weibull parameters based on (9) - see Figure 5. The highest posterior density now corresponds to $\alpha=52.1$, and $\beta=2.05$. 


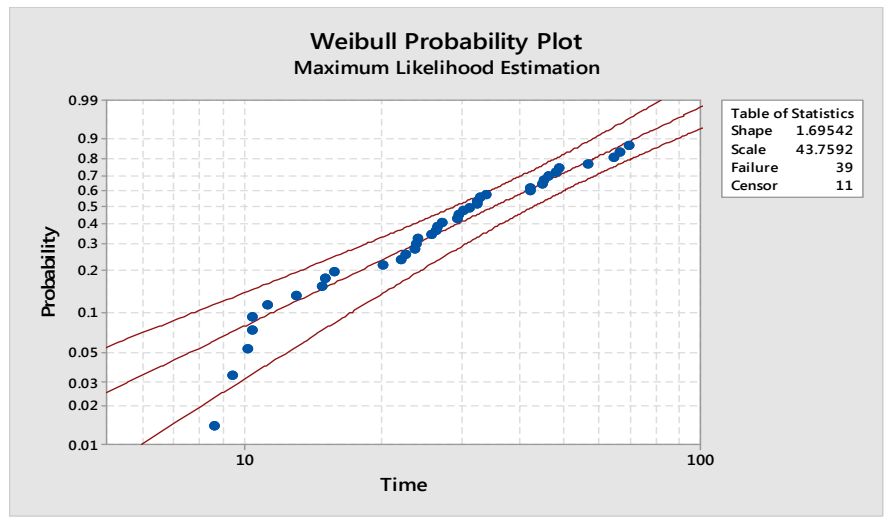

Figure 1: A random Weibull sample to be used for constructing the joint prior distribution of Weibull parameters.

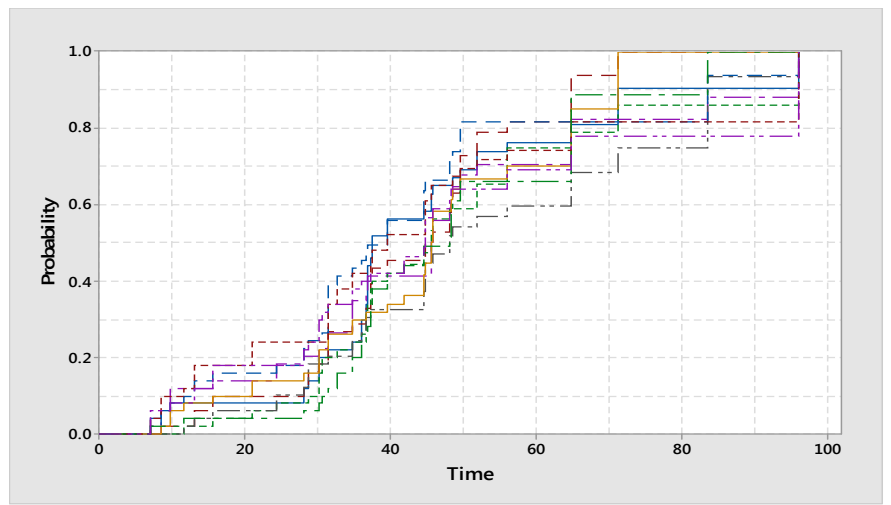

Figure 2: Some bootstrap samples from the Weibull sample in Fig. 1.

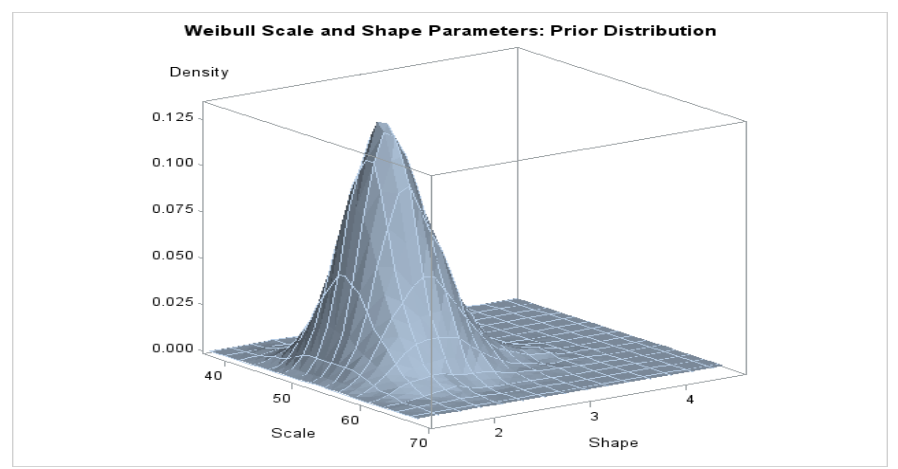

Figure 3: The joint prior distribution of Weibull parameters constructed based on 10,000 bootstrap simulations from the Weibull sample in Fig. 1. 


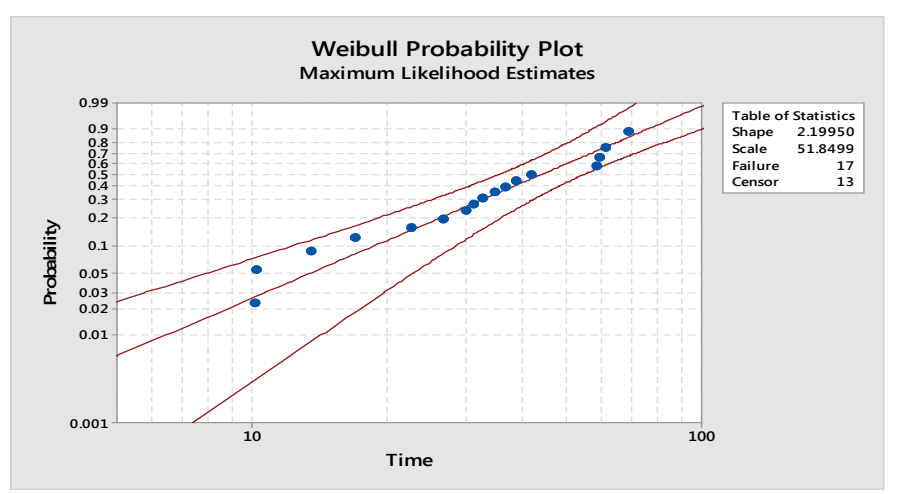

Figure 4: A random Weibull sample to be used as current data for constructing the joint posterior distribution of Weibull parameters.

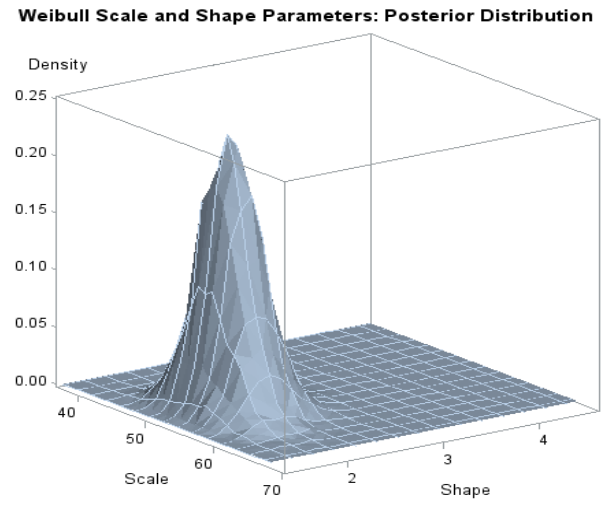

Figure 5: The joint posterior distribution of Weibull parameters.

Finally, using (10)-(12), we obtain the posterior Weibull reliability function with respective credibility intervals - see Figure 6.

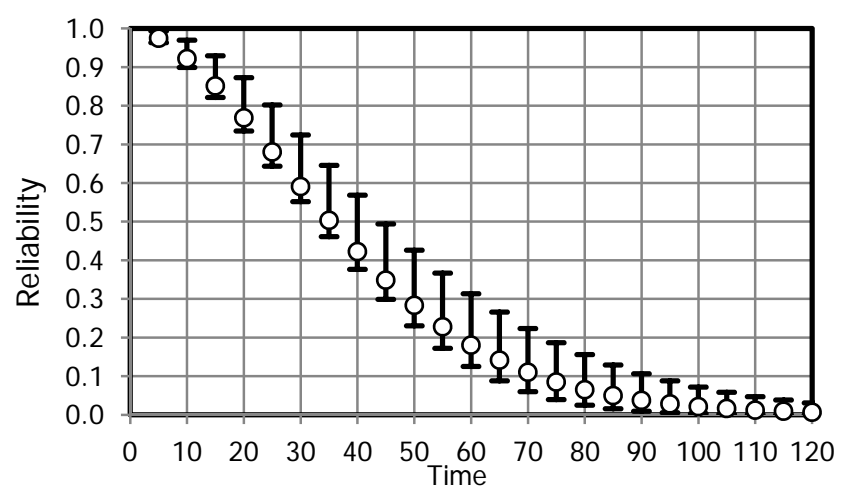

Figure 6: The posterior Weibull reliability functions with 95\% two-sided credibility intervals. 


\section{Concluding Remarks}

In practical reliability engineering, construction of the joint prior PDF for the parameters of the underlying lifetime distribution is a challenging task. This paper presented a simple procedure of Bayesian estimation of the Weibull distribution based on a single random sample characterizing prior data and a single random sample characterizing current data.

The Weibull distribution is discussed as an illustrative example, but the proposed procedure can be extended to any parametric lifetime distribution, not necessarily limited to the location-scale family. Moreover, it can even be used with empirical distributions without assuming any parametric structure.

\section{Acknowledgement}

We would like to acknowledge the review and valuable suggestions of Dr. Mark Kaminskiy of NASA Goddard Spaceflight Center, Greenbelt, MD, USA.

\section{References}

[1]. Kaminsky, M.P., and V.V. Krivtsov. A Simple Procedure for Bayesian Estimation of Weibull Distribution, IEEE Transactions on Reliability, 2005; 54(2): 612-616.

[2]. Canavos, G.C., and C.P. Tsokos. (1973). Bayesian estimation of life parameters in the Weibull distribution, Operations Research, 1973; 21: 755-763.

[3]. Meeker, W.Q., and L.A. Escobar, Statistical Methods for Reliability Data, John Wiley \& Sons, New York, 1998.

[4]. Google Scholar Citations, http://scholar.google.com/intl/en/scholar/citations.html, retrieved: 11/2/2016.

[5]. SAS Institute Inc. New Features in JMP ${ }^{\circledR} 11$. Cary, NC, 2013.

Vasiliy Krivtsov is the Director of Reliability \& Safety Analytics at the Ford Motor Company. He also holds the position of Adjunct Professor at the University of Maryland, where he teaches a graduate course on advanced reliability data analysis. Krivtsov has earned a $\mathrm{PhD}$ degree in Electrical Engineering from Kharkov Polytechnic Institute (Ukraine) and a $\mathrm{PhD}$ in Reliability Engineering from the University of Maryland, USA. Krivtsov is the author and co-author of over 60 professional publications, including 2 books on Reliability Engineering and Risk Analysis, 9 patented inventions and 6 trade secret inventions on statistical algorithms for Ford. He is a Vice Chair of the International Reliability Symposium $\left(\right.$ RAMS $\left.^{\circledR}\right)$ Tutorials Committee and a Senior Member of IEEE. Prior to Ford, Krivtsov held the position of Associate Professor of Electrical Engineering in Ukraine, and that of Graduate Research Scientist at the University of Maryland Center for Reliability Engineering. Further information on Dr. Krivtsov's professional activity is available at www.krivtsov.net.

Michael Frankstein is a Senior Research Engineer with the Ford Motor Company. He holds an M.S. degree in Mathematics from the Moscow State University (Russia) and a Ph.D. in Reliability Engineering from the National Scientific Research Institute in Automotive Industry (Moscow, Russia). Dr. Frankstein is the author and co-author of more than 20 professional publications and one patented invention. Prior to Ford, Dr. Frankstein held multiple research and development positions including that of a Senior Scientist with the Automotive Research and Development Center (Moscow, Russia). 